\title{
EXPERIMENTACIÓN EN TORNO AL PROYECTO DE INVESTIGACIÓN SOBRE EL YACIMIENTO NEOLÍTICO DE LA DRAGA (BANYOLES- GIRONA)
}

Experimentation around the research project on the neolithic site of la Draga (BanyolesGirona)

\author{
Antoni Palomo ${ }^{1}$, Xavier Terradas ${ }^{2}$, Raquel Piqué ${ }^{3}$, Oriol López-Bultó ${ }^{4}$, Miriam de Diego ${ }^{5}$, \\ Ignacio Clemente ${ }^{6}$ \\ https://doi.org/10.15366/baexuam2020.14.005
}

\section{RESUMEN}

En torno al proyecto de investigación del yacimiento neolítico lacustre de la Draga (Banyoles-Girona) se llevan a cabo desde hace más de una década programas experimentales centrados en diversas cuestiones tecno funcionales. La extraordinaria conservación de los materiales arqueológicos realizados tanto en materias inorgánicas como orgánicas han permitido desarrollar experimentos que han replicado procesos técnicos de producción y utilización de diferentes herramientas y artefactos de hueso, madera y piedra, utilizados en diferentes actividades, como el trabajo de la madera y la producción textil. El proyecto experimental de la Draga se plantea como una herramienta holística de investigación arqueológica, de formación académica y de divulgación para todos los públicos.

\section{Palabras claves}

Neolítico, tecnofuncional, materia orgánica, lacustre, formación académica, difusión

\begin{abstract}
Experimental programs focused on various techno-functional issues have been carried out for more than a decade around the research project of the La Draga lacustrine neolithic site (Banyoles-Girona).The extraordinary conservation of archaeological materials made from both inorganic and organic materials has allowed the development of programs that have replicated technical processes of production and use of different tools and artifacts of bone, wood, and stone, used in different activities, such as woodworking and textile production. The Draga experimental project is proposed as a holistic tool for archaeological research, academic training, and dissemination for all audiences.
\end{abstract}

\section{Keywords}

Neolithic, techno-functional, organic matter, lacustrine, academic training, outreach

\footnotetext{
${ }^{1}$ Museu d'Arqueologia de Catalunya. antoni.palomo@gencat.cat ORCID 0000-0001-9954-7310

${ }^{2}$ Consejo Superior de Investigaciones Científicas-IMF ORCID 0000-0002-8000-5607

${ }^{3}$ Universidat Autònoma de Barcelona ORCID 0000-0002-8253-6874

${ }^{4}$ Universidat Autònoma de Barcelona ORCID 0000-0001-5327-1881

${ }^{5}$ Universidat Autònoma de Barcelona

${ }^{6}$ Consejo Superior de Investigaciones Científicas-IMF ORCID 0000-0002-3190-215X
} 


\section{El yacimiento de la Draga}

La Draga (Banyoles, Pla de l'Estany) es el único yacimiento neolítico lacustre conocido en la Península Ibérica y uno de los más antiguos con estas características en el ámbito Mediterráneo, superando su cronología en más de un millar de años a la de los conocidos yacimientos lacustres del centro de Europa. A nivel regional se sitúa en el Neolítico antiguo (facies con cerámicas impresas cardiales); periodo bien conocido en la zona costera que se extiende entre Provenza y Andalucía. Las dataciones radiométricas obtenidas por carbono 14 enmarcan la vida del poblado en la segunda mitad del VI milenio AC, con una fase inicial entre 5300 a $5100 \mathrm{cal}$ AC y una fase final entre 5.100-4900 cal AC (Palomo et al. 2014; Bogdanovic et al. 2015; Andreaki et al. 2020).

La Draga se emplaza en la orilla oriental del lago de Banyoles, a 170 m.s.n.m, a $40 \mathrm{~km}$ de la actual línea de costa mediterránea y a $50 \mathrm{~km}$ al sur de los Pirineos. El lago tiene forma de figura de ocho y el asentamiento se situó en el punto más estrecho de su orilla este (Figura. 1).

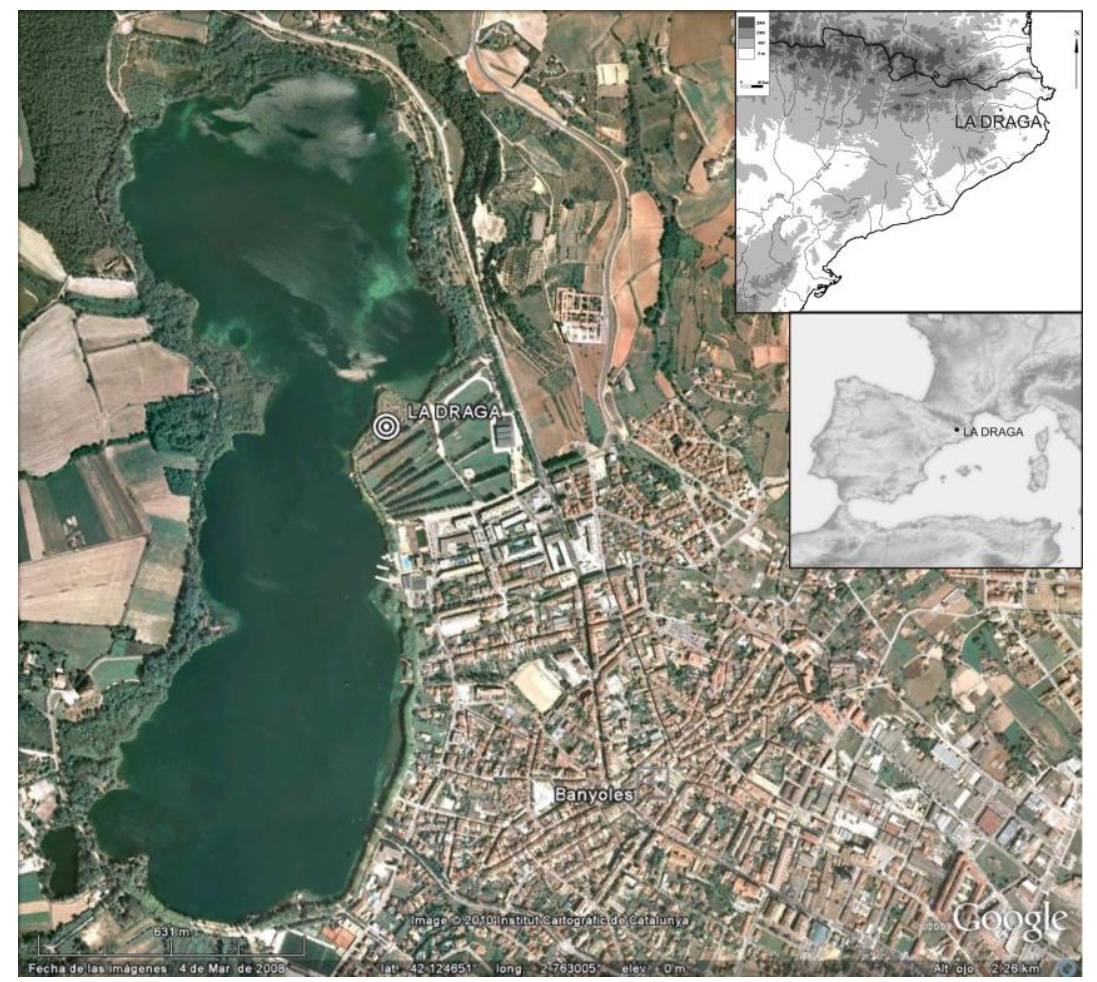

Figura 1. Situación del yacimiento de la Draga (Banyoles-Girona)

Desde su descubrimiento en el año 1990, cuando se iniciaron los trabajos arqueológicos, ya se determinó su singularidad y potencialidad para entender las formas de vida de las primeras comunidades agrícolas y ganaderas del noreste peninsular. Los trabajos arqueológicos realizados de forma prácticamente ininterrumpida han permitido alcanzar un considerable impacto sobre la investigación del proceso de neolitización. La Draga se ha convertido en un yacimiento capital para comprender el proceso de implantación de las comunidades neolíticas en el área mediterránea y la difusión de sus bases económicas hacia tierras interiores.

El yacimiento de la Draga es excepcional por varios motivos. En primer lugar, por su antigüedad, que lo sitúa entre los más antiguos del noreste peninsular. En segundo lugar, 
por el hecho de tratarse de un asentamiento al aire libre con una cierta continuidad en la ocupación del sitio y una estratigrafía bien conservada, sin alteraciones tafonómicas notables. Por último, y quizás lo más remarcable, por sus condiciones excelentes de conservación. Una parte de los estratos arqueológicos se encuentran en el nivel de aguas freáticas lo que ha propiciado unas condiciones anaeróbicas especiales. Esta situación ha permitido una extraordinaria conservación de los restos orgánicos y materias vegetales (madera y fibras vegetales) (Bosch/Chinchilla/Tarrús 2006).

Es muy destacable la ingente documentación generada a partir de las estructuras de madera construidas, de las que hasta el momento se han recuperado 1.300 postes verticales de madera y cientos de elementos horizontales que formaban parte de las cabañas del poblado. La Draga también cuenta con uno de los conjuntos de instrumentos del Neolítico antiguo europeo más importantes y variados realizados en madera y / o fibra vegetal. Se trata de 177 útiles entre los que cabe destacar, por ejemplo, los tres arcos más antiguos del Neolítico europeo (Piqué et al. 2015), mangos de hoces (Palomo et al. 2011), mangos de azuela (Bosch et al. 2008), cuerdas (Piqué et al. 2018), cestos (Romero et al. en prensa), recipientes de madera y cucharones, palos cavadores (López Bultó et al. 2020), peines y husos (de Diego et al. 2017, 2018), además de objetos de uso desconocido (Figura 2).

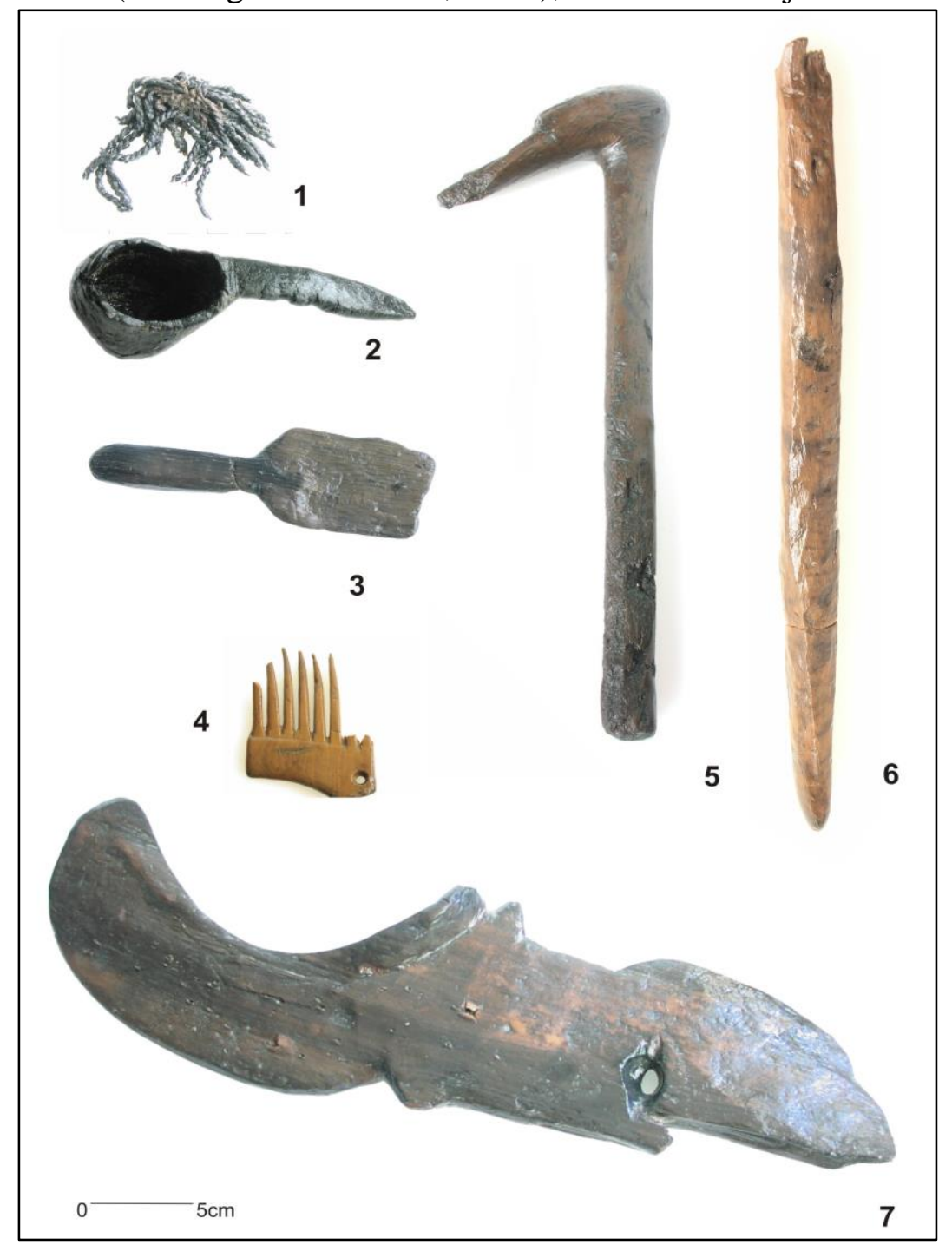

Figura 2. Diferentes herramientas y mangos realizados con madera y fibras vegetales. 1. Cuerda de arco, 2. Cucharon, 3. Pequeña pala, 4. Peine, 5. Mango de azuela, 6. Pala cavador y 7. Gancho de madera. 
Por otra parte, la buena conservación de los restos arqueológicos no se limita a la materia orgánica, también destaca la buena preservación de la estratigrafía, de las estructuras construidas en piedra y de todo tipo de artefactos. En este sentido, y por poner un ejemplo, cabe señalar que la Draga ha proporcionado los conjuntos de restos arqueozoológicos, antracológicos y carpológicos mejor conservados para este periodo en el NE peninsular (Antolín/Buxó 2011; Saña, 2011).

\section{El porqué de la experimentación y el marco de su desarrollo}

Las características del yacimiento de la Draga son idóneas para poder desarrollar programas de investigación donde la experimentación tenga un papel relevante y pueda responder a preguntas que no siempre tienen respuesta. Cabe remarcar que se trata de un poblado con una horquilla cronológica relativamente amplia, de prácticamente 400 años, con una vasta área excavada próxima a los $1000 \mathrm{~m} 2$. Los trabajos arqueológicos llevados a cabo durante casi tres décadas han permitido documentar con precisión una gran diversidad de procesos técnicos que se desarrollaron in situ en el asentamiento. Además, es uno de los pocos casos donde se pueden integrar los trabajos experimentales de carácter tecnofuncional sobre materias orgánicas e inorgánicas y así obtener una aproximación lo más precisa posible a la tecnología de las primeras comunidades neolíticas.

Otro de los aspectos a destacar es que una buena parte del amplio equipo que trabaja en la Draga está formado por investigadores e instituciones que han fundamentado su trabajo en la experimentación arqueológica. En este sentido, cabe destacar el papel del CSIC-IMF en la implementación de trabajos en relación a las producciones con materias abióticas, y la $\mathrm{UAB}$ con restos bióticos.

Para nosotros, la experimentación es inherente al método científico y una parte integral del mismo (Baena/Terradas 2005), ya que entendemos que es la base de la investigación científica y también, por lo tanto, de la investigación arqueológica. La investigación en la Draga implica el uso de la experimentación como una forma de contrastar las hipótesis formuladas a partir de los diferentes análisis realizados previamente.

La investigación experimental se ha centrado en replicar los procesos técnicos de producción y utilización de diferentes herramientas y artefactos de hueso, madera y piedra, relacionados con un gran número de actividades, como pueden ser el trabajo de la madera, la producción textil... (Palomo, 2012; López-Bultó, 2015, Palomo et al., 2017., De Diego et al 2017 a y b, 2018; Clemente et al. 2014... ).

Con este fin se han reproducido diferentes tipos de herramientas para responder diferentes cuestiones en relación a su utilización (efectividad, ergonomía, gestualidad...), así como construir una colección de referencia de rastros tecnológicos y funcionales cuyos elementos puedan ser comparables con los artefactos arqueológicos (Mozota et al. 2018). La identificación de las técnicas de manufactura se ha logrado mediante el análisis de los estigmas técnicos que aparecen en los artefactos arqueológicos de piedra, de madera, de hueso, de concha..., y la comparación con los producidos durante la experimentación. De esta manera, hemos podido identificar qué herramientas se usaron durante la manufactura 
de artefactos y obtener así una mejor comprensión de los procesos de producción y uso de las herramientas.

El proyecto experimental de la Draga se plantea como una herramienta holística de investigación arqueológica, de formación y divulgación académica y también como herramienta pedagógica que permita la difusión de los resultados a todos los públicos. En este sentido, el equipo de la Draga ha realizado y participado en seminarios especializados sobre experimentación en relación a las comunidades neolíticas ${ }^{7}$, organizando congresos (III Congreso Internacional de Arqueología experimental, Banyoles 2011) (Palomo, Piqué, Terradas 2013), participado en congresos especializados nacionales e internacionales y realizado propuestas de transferencia científica para el público general. En este sentido, cabe destacar que los resultados conseguidos de los trabajos experimentales han tenido una aplicabilidad en las propuestas didácticas que se ofertan desde el Parc Arqueològic del Poblat Neolític de la Draga (Buch, Comellas, Palomo 2011, 2013) desde su inauguración en el año 2003. Este hecho ha permitido que el parque arqueológico se haya convertido como un referente didáctico en Catalunya para entender la vida de las primeras sociedades agrícolas y ganaderas.

Los trabajos experimentales realizados hasta el momento se han integrado en diversos proyectos de investigación y han dado como fruto diversos trabajos de investigación como tesis doctorales y trabajos de fin de máster, además de contribuir a una producción bibliográfica relativamente importante que mostramos parcialmente en este artículo a partir de diversas citas.

\section{Experimentación sobre madera}

Sin duda las experimentaciones sobre herramientas de madera son las más singulares de las realizadas y han permitido reconocer los procesos fundamentales de producción, así como también de su uso (Palomo 2012, López-Bultó, 2015, López-Bultó et al. 2020). En especial, hemos desarrollado programas experimentales para la reproducción y utilización de herramientas relacionadas con la caza, la carpintería y las prácticas agrícolas.

La buena conservación de los objetos y su diversidad permiten plantear el estudio de los procesos técnicos vinculados a la transformación de la madera en la Draga desde una perspectiva completamente diferente a la de los yacimientos tradicionales. Contamos con los productos finales, herramientas en proceso de elaboración, residuos generados durante el procesado de la madera y los propios instrumentos que podrían haber sido utilizados para este fin.

Los estudios tecnológicos previos de los propios objetos de madera y de los instrumentos de piedra han permitido documentar cuestiones relacionadas con las materias primas utilizadas, características de los soportes, desbastado, tipos de acabados, etc. (Bosch et al 2006) y generar hipótesis sobre cómo se llevó a cabo la transformación de la madera. Sin

\footnotetext{
${ }^{7}$ En otros relacionados con la fabricación y uso de herramientas de piedra pulida, de madera, técnicas agrícolas y elaboración de herramientas de fibras vegetales. Por ejemplo.: https:/www.uab.cat/web/detalle-denoticia/seminario-de-arqueologia-experimental-en-la-draga1345697212752.html?noticiaid $=1345684686840$
} 
embargo, desde nuestro punto de vista, sólo es posible contrastar dichas hipótesis a partir del trabajo experimental.

Con este objetivo, hemos llevado a cabo un intenso programa de experimentación centrado en desarrollar una metodología que permita determinar los procesos de producción de los objetos de madera. La experimentación se ha centrado en buscar indicadores que permitan la identificación del soporte empleado (tronco, rama), el proceso de desbastado del tronco y los acabados. En definitiva, se ha tratado de reconocer y reproducir las técnicas empleadas en el proceso de elaboración de los objetos de madera.

El programa experimental se ha centrado en producir material de referencia en el que sea posible observar la diversidad de huellas resultado del proceso de elaboración de los objetos. Los gestos y acciones necesarios para la réplica de los objetos han surgido de las hipótesis surgidas mediante la observación de sus características. Así, este proceso debe permitir establecer la idoneidad de los criterios utilizados para su estudio tecnológico. La experimentación se ha orientado también a contrastar la eficacia de los instrumentos arqueológicos que presumiblemente se utilizaron en el trabajo de la madera (lascas y láminas de sílex, azuelas con hoja de piedra, así como cuñas de madera) (Figura 3).

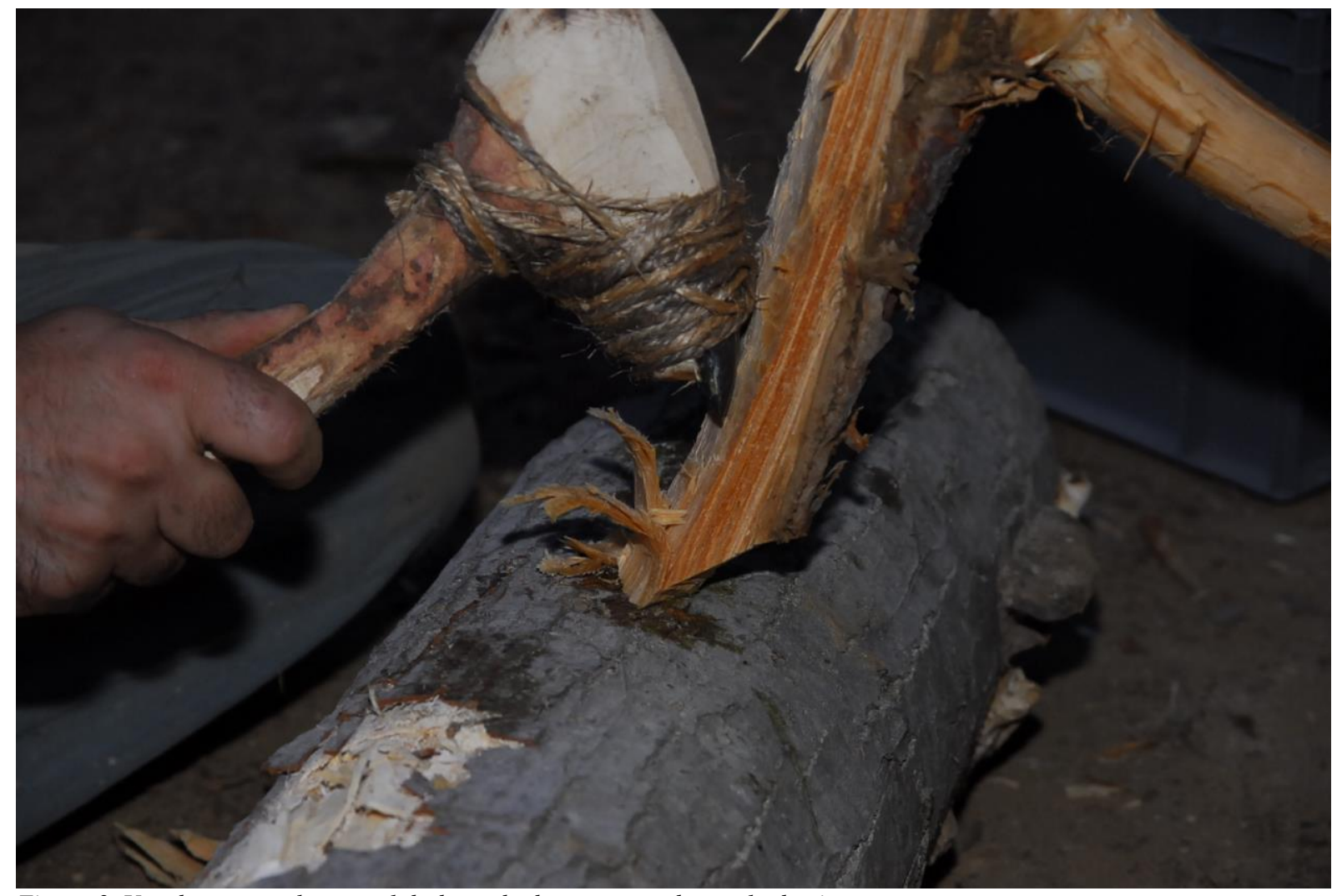

Figura 3. Uso de una azuela para el desbastado de un mango de azuela de pino.

Asimismo, hemos procedido a replicar instrumentos concretos de la Draga para contrastar las hipótesis formuladas acerca de sus procesos de manufactura. Los instrumentos escogidos fueron los mejor representados en el conjunto arqueológico: los mangos de azuela (Bosch et al. 2006, 2008), los palos cavadores (López-Bultó 2015, López-Bultó et al. 2020, López-Bultó et al on press, 2020), los mangos de hoz (Palomo et al. 2011) y los 
arcos (Piqué et al. 2015). Lascas y láminas de sílex, así como azuelas con hoja pulida de corneana y cuñas de madera fueron los instrumentos utilizados para la experimentación (Palomo et al. 2011b, 2011c, Palomo, 2012).

Los procesos fundamentales que se han determinado mediante el análisis tecnológico y su corroboración experimental son los siguientes (Figura 4):
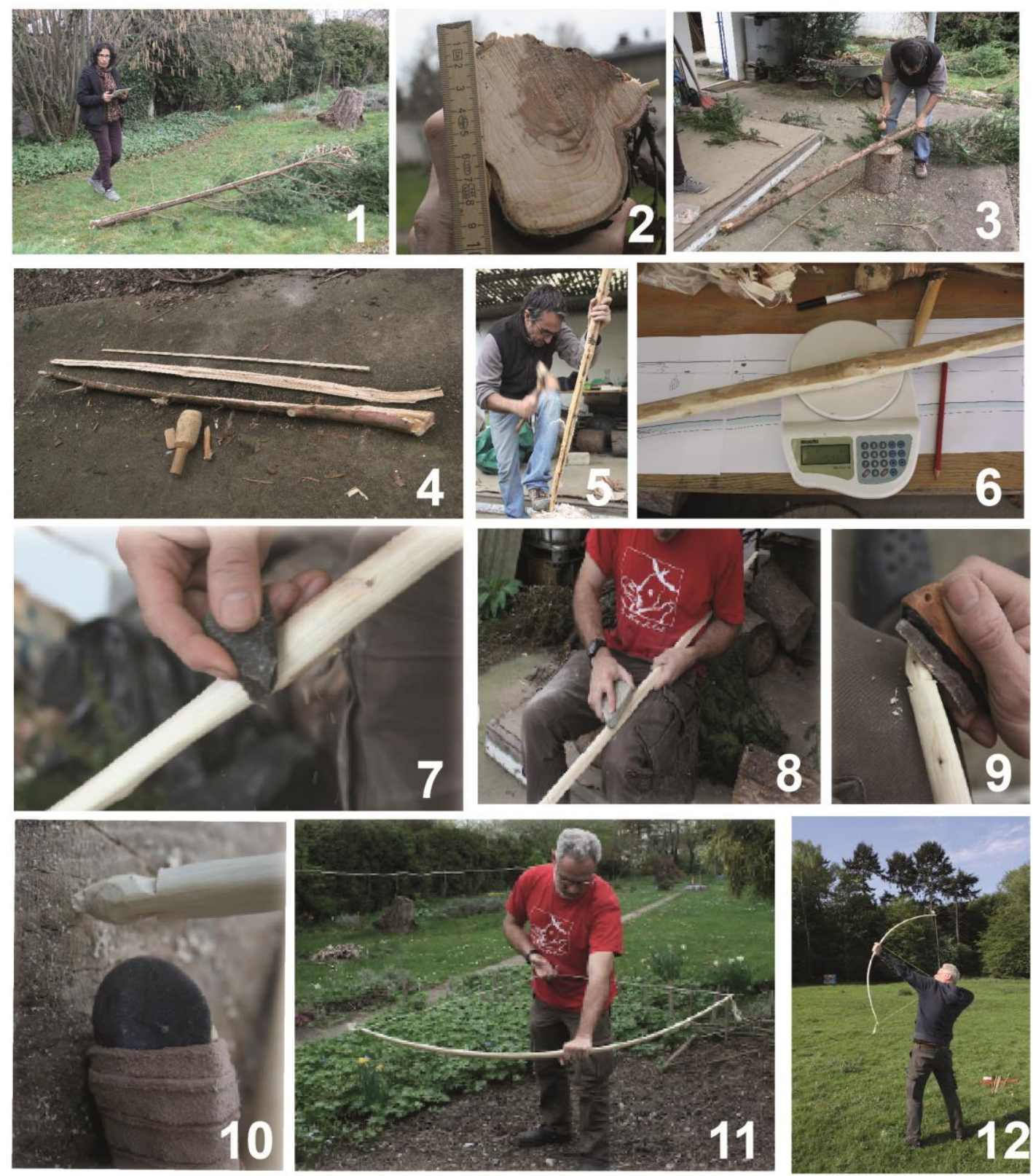

Figura 4. Proceso de elaboración de una réplica de un arco usando los procesos fundamentales del trabajo de la madera: 1. Obtención de la materia, 2. Detalle de la sección, 3 y 5. Desbastado, 4. Detalle de la segmentación realizada mediante cuñas de madera, 6. Pesado de la madera para controlar el secado, 7. Acabado mediante una lasca de sílex, 8. Acabado/pulido mediante una arenisca, 9. Realización de la muesca de encaje de la cuerda, 11 y 12. Tensado y prueba del arco. 
-La obtención de la materia prima:

Los análisis taxonómicos revelaron un uso específico de los taxones según el elemento u objeto que se pretendía elaborar (Bosch et al.2006). Este estudio sugería también que los soportes (tronco o rama) fueron seleccionados según sus formas o dimensiones, aprovechando a menudo las formas originales que estos tenían para determinados elaborar determinados objetos. La experimentación corroboró la idoneidad de los tipos de madera seleccionados, tanto la especie como la parte del árbol/arbusto, para la fabricación de las herramientas y los mangos. Por ejemplo, una rama y la parte del tronco de pino o roble de la que surge es el soporte ideal para la confección de un mango de azuela. Asimismo, una rama o tronco rectilíneo de boj es la base ideal para la confección de palos cavadores.

Dadas las características de los utensilios de piedra pulida localizados en la Draga y los mangos acodados, se puede afirmar que la tala de los árboles o arbustos se realizó mediante el uso exclusivo de azuelas. La experimentación ha permitido corroborar la eficiencia de este instrumento para la tala del tronco y corte de ramas durante la fase de obtención de la materia prima. El rebajado perimetral y posterior fractura es la técnica documentada en la Draga para talar los troncos y ramas.

La materia prima debió llegar al poblado en forma de rama o tronco, donde se debía transformar para darle la morfología deseada. En la Draga, se han localizado algunos restos de talla de desbaste de la madera y objetos de madera en proceso de elaboración que corroboran que los trabajos de carpintería se llevaron a cabo en el asentamiento, por lo menos en el caso de algunas de las especies identificadas.

-Extracción de la corteza:

Algunos de los utensilios y mangos no conservan la corteza, lo que nos demuestra que existía la voluntad de extraerla. La operación se podría llevar a cabo previamente a la segmentación o bien en cualquier momento del proceso de producción. El estudio traceológico (Gibaja, 2000) hecho sobre el utillaje lítico documentado en la Draga, esencialmente lascas y láminas de sílex, nos ha permitido reconocer un conjunto de instrumentos que fueron destinados al trabajo de la madera, la extracción de la corteza pudo ser una de las acciones realizadas con estos instrumentos. También se ha podido determinar la utilización de valvas como herramienta de trabajo para procesar la madera en acciones como las de raspado de la corteza (Palomo et al. 2013)

\section{-Segmentación.}

En una buena parte de los palos cavadores y palos apuntados, los mangos de azuela, arcos, algún tipo de hoz, así como en parte de la preparación de algunos elementos arquitectónicos (tablones, ...), el proceso de fabricación pasa por hendir radialmente el tronco o la rama para obtener segmentos. Esta técnica se documenta en la elaboración de los palos cavadores, que se confeccionaron mayoritariamente a partir de un segmento de un cuarto de tronco, de los mangos de las azuelas, hoces y de los arcos. Experimentalmente hemos podido constatar que el hendido del tronco se puede efectuar utilizando elementos que actúan como cuñas. 
Entre los utensilios líticos tallados de La Draga las piezas astilladas son los que se han podido utilizar como cuña para segmentar la madera. Los bordes presentan importantes muescas bifaciales, lo que demuestra el fuerte impacto que ha sufrido la parte activa durante su utilización. Nuestros experimentos han permitido reconocer que este tipo de instrumentos tienen un comportamiento muy eficaz para esta actividad, aunque, según la dureza de la madera, se fracturan con bastante facilidad (Gibaja et al. 2007, Palomo 2012). En la Draga, este tipo de utensilio es relativamente abundante, con un $10 \%$ de los útiles retocados (Palomo 2011d) y en el Neolítico antiguo son relativamente presentes en el noreste peninsular (Palomo, 2012)

Por otra parte, otro utillaje empleado para la segmentación de los soportes de madera son las cuñas de madera de boj, de las cuales se han recuperado dos ejemplares en el yacimiento (Bosch/Chinchilla/Tarrús 2006; Palomo 2012, 108). El boj es una madera resistente, dura y, si se generan filos agudos, muy penetrante en maderas verdes, incluso si son duras como el roble. Los experimentos realizados nos confirman su eficacia para hendir longitudinalmente la madera de tejo para confeccionar arcos, el pino para la realización de mangos de azuela y el boj para la confección de palos cavadores.

- El desbastado.

Los utensilios y mangos de madera analizados presentan pequeñas trazas simétricas y grandes facetas de extracción de materia que permiten, por un lado, reducir el volumen de la materia y, por otro, darle la forma deseada. La experimentación ha permitido confirmar que las pequeñas trazas son el resultado del uso de una azuela, y las facetas grandes del proceso de hendido llevado a cabo mediante cuñas.

\section{- Acabado.}

Los elementos arquitectónicos, así como algunos de los objetos de madera de la Draga no presentan ningún tipo acabado más allá de los estrictamente funcionales. Sin embargo, en algunos de los utensilios y los mangos no son visibles las facetas resultantes del trabajo con azuela ni las grandes facetas del hendido debido a que la superficie ha sido totalmente regularizada o pulida. La regularización, el adelgazamiento mediante el raspado de la superficie, se podría hacer con un utensilio lítico tallado de las mismas características que los empleados en la extracción de la corteza y que hemos descrito anteriormente, ya sean elaborados con sílex o conchas de la especie Donax sp. (Clemente/Cuenca 2011, Clemente et al. 2014; Palomo et al. 2013).

Hemos interpretado que el pulido de la superficie se debía realizar con la ayuda de un gres o arenisca, muy abundante en el contexto arqueológico, que permitía que la superficie se homogeneizara. Sin embargo, somos conscientes de que el uso mismo de los utensilios y mangos podría resultar en un pulimento accidental. La discriminación entre las huellas tecnológicas y funcionales se está tratando desde una perspectiva experimental, que ya ha dado resultados positivos y que esperamos se acrecentarán en un futuro próximo.

Algunos instrumentos, como los mangos de hoz, presentan una o más ranuras para el encaje de la hoja de sílex. Estas ranuras se podrían haber realizado mediante un utensilio lítico tallado. De hecho, el estudio de las huellas de uso (Gibaja, 2000) de los instrumentos 
líticos evidencia el trabajo de corte en lascas o láminas. En este caso, los bordes de estos instrumentos líticos no sólo no están retocados, sino que, además, muestran filos más agudos que los utilizados para raspar. En este sentido, entre los utensilios líticos utilizados para cortar, sobresalen aquellos que tienen un ángulo agudo, que puede oscilar entre los $20^{\circ} \mathrm{y} \operatorname{los} 40^{\circ}$.

\section{La producción lítica tallada y su uso}

Nuestro trabajo también se ha centrado en el estudio de la producción y el uso de herramientas de piedra talladas documentadas en la Draga, dónde se ha recuperado una amplia gama de herramientas líticas hechas de rocas silíceas, principalmente de sílex. Esta producción lítica se caracteriza por la producción de soportes laminares utilizados principalmente como elementos activos en hoces, proyectiles y taladros. Por otra parte, varios candiles de asta de ciervo arqueológicos recuperados en la Draga se han interpretado como un conjunto de útiles de talla utilizados como elementos intermedios en la percusión indirecta. En base a los datos que revelaron los diferentes análisis tecnológicos de estos materiales se desarrolló un programa experimental para determinar la técnica utilizada en la producción laminar (Palomo 2011d, 2012) (Figura 5).
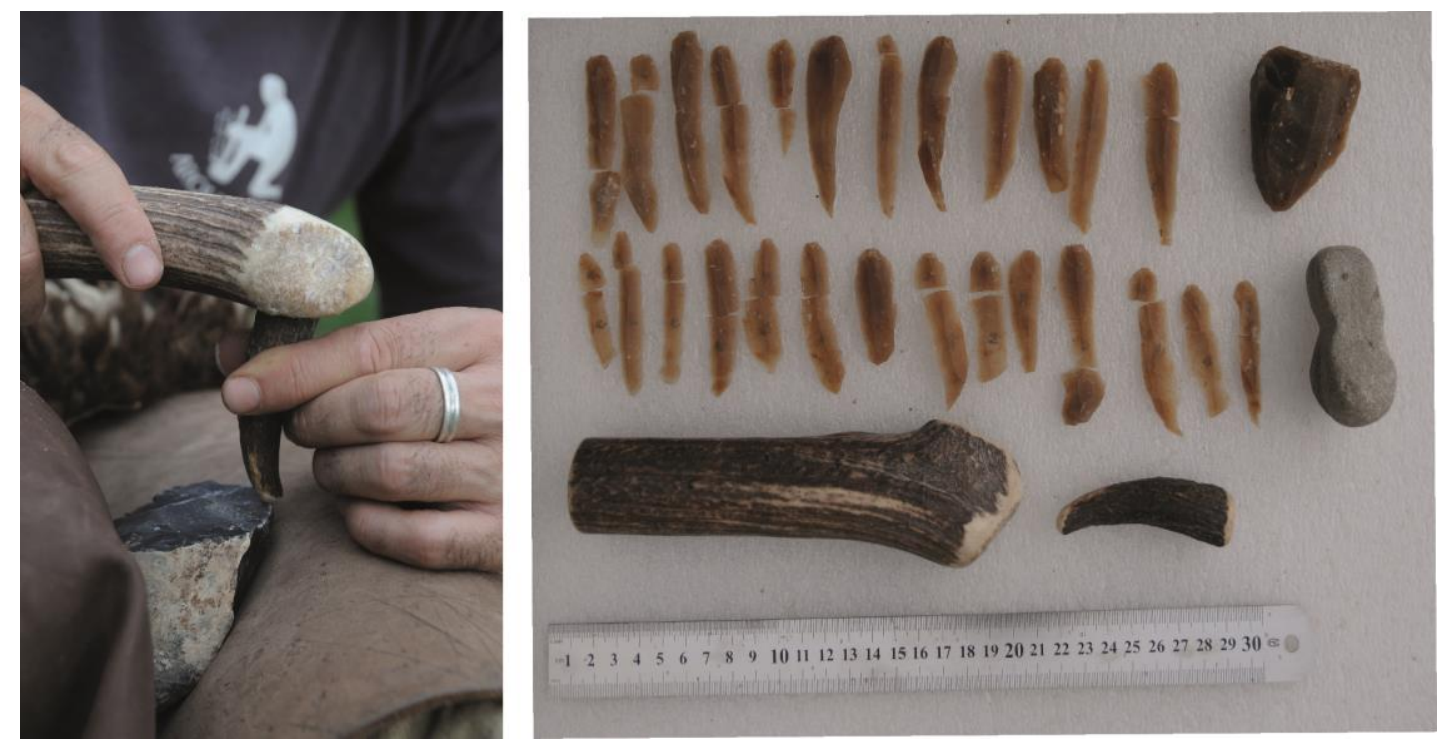

Figura 5. Experimentación del uso de la técnica de la percusión indirecta, productos y herramientas utilizadas.

Los experimentos se centraron en dos aspectos principales. Por una parte, la determinación de la técnica utilizada en la producción laminar. Por otra, la contrastación de la hipótesis de que los candiles de asta de ciervo eran punteros utilizados en la talla por percusión indirecta.

Los resultados obtenidos permitieron corroborar que una parte de la producción plena laminar presenta unas características morfotécnicas atribuibles al uso de la técnica de la percusión indirecta. Por otra parte, los estudios de los punteros de asta de ciervo determinaron que éstas presentaban trazas y deformaciones generadas al utilizarlos como elementos intermedios en el proceso de producción lítica.

Finalmente, diversos experimentos nos han permitido una aproximación a la función de diversos tipos de útiles; entre los cuales cabe destacar los proyectiles geométricos (Figura 
6) (Gibaja/Palomo 2004, 2006; Gibaja, Palomo, Armengol 2011; Palomo 2012) y el uso de las hoces (Palomo et al. 2011a).

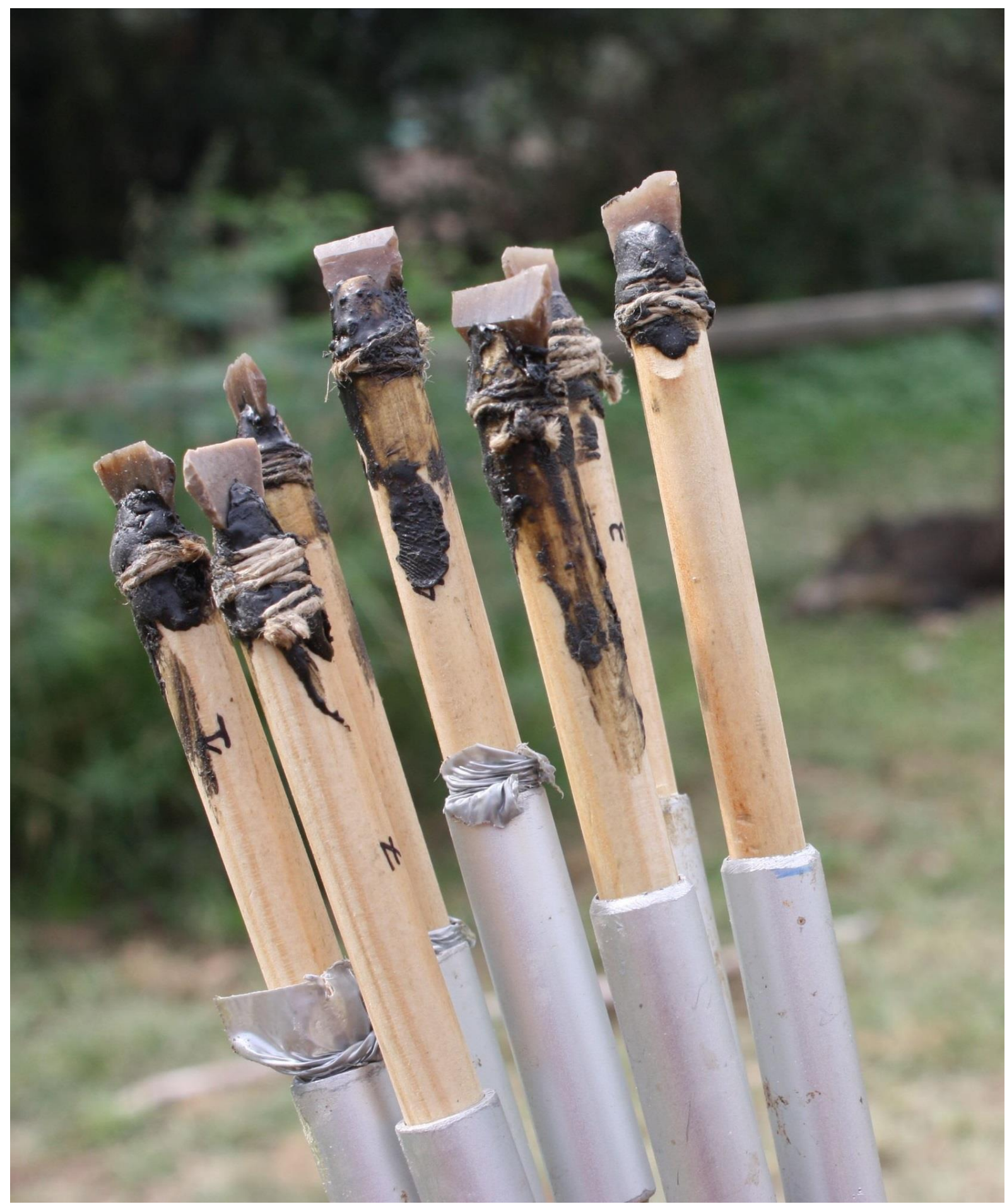

Figura 6. Puntas geométricas preparadas para su uso experimental.

\section{Tecnología textil}

La tecnología textil es otro de los procesos tecnológicos sobre el cual hemos desarrollado un programa experimental. Nuestro objetivo ha sido determinar si algunos instrumentos 
recuperados en la Draga pudieron haber sido utilizados en procesos textiles, tal como sugería su morfología. Entre los artefactos relacionados con el trabajo textil se encuentran algunos que presentan forma de huso, de lanzadera y de peine, elaborados todos ellos en madera; así como agujas, punzones y otras herramientas de hueso. Todos estos instrumentos son similares a los utilizados por las sociedades modernas y antiguas en los procesos de tejido e hilado como parte de la producción textil tradicional. El objetivo de la experimentación se ha centrado en generar una colección de referencia de trazas de usodesgaste para poder ser utilizados como elemento de comparación en el estudio del material arqueológico (Figura 7).
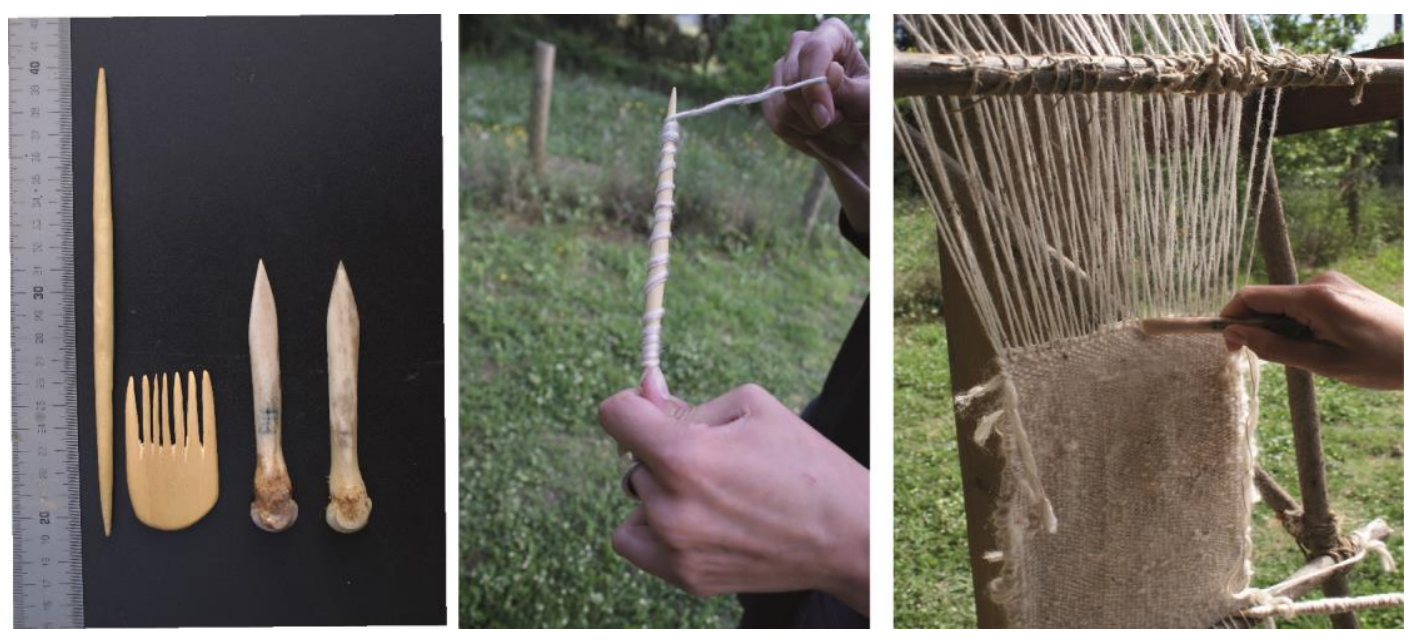

Figura 7. De derecha a izquierda: huso y peine de madera de boj y punzones experimentales, utilización del buso para hilar y prensado de la trama mediante un punzón de hueso.

Las acciones realizadas durante la experimentación del proceso de producción textil fueron:

- Uso de peines de madera para peinar y cardar la lana.

- Uso de una lanzadera elaborada en madera para tejer.

- Uso de un huso de madera para hilar.

- Uso de punzones de hueso empleados durante el tejido como prensadores de trama para aumentar la densidad textil, y también para seleccionar y separar los hilos de la urdimbre.

Los resultados de las experimentaciones permiten defender la hipótesis que en el poblado de la Draga existen datos indirectos, ya que no se conservan los tejidos, que sostienen la hipótesis de la implementación de una tecnología textil incipiente con fibras vegetales (Diego et al. 2017 a y b y 2018) durante el Neolítico antiguo.

\section{Reflexiones finales}

Prácticamente 20 años después de iniciar los primeros trabajos experimentales en el yacimiento de la Draga podemos constatar la utilidad de algunas propuestas experimentales para resolver cuestiones tecnofuncionales. Además, la aplicabilidad de dichas aproximaciones en el marco de propuestas pedagógicas ha sido sobradamente contrastada en el caso del Poblat Neolític de La Draga. Por otra parte, los resultados de los diferentes trabajos han sido ampliamente publicados en una extensa bibliografía (que ya hemos expuesto parcialmente) tanto en el ámbito estatal como internacional, y defendidos en diversos trabajos académicos de investigación. En definitiva, la experimentación 
constituye un elemento fundamental en la dinámica científica de nuestro grupo de investigación que permite abordar las problemáticas más diversas, habiéndose mostrado como un medio de amplio alcance en la contrastación y/o refutación de hipótesis.

Después de décadas de trabajo, las propuestas experimentales han pasado de ser una anécdota en la investigación arqueológica a presentarse como una normalidad necesaria que ha ido calando en diversos ámbitos de la investigación arqueológica y la formación académica. En este sentido, integrantes de nuestro grupo de investigación participan activamente en programas académicos universitarios dónde imparten contenidos relacionados con la experimentación, en la organización de seminarios de tecnología y en la tutoría de trabajos de investigación donde la experimentación se presenta como un activo metodológico imprescindible. En definitiva, proyectos como el de la Draga (entre otros) han impulsado que la arqueología experimental se haya convertido en un elemento primordial en la investigación fundamental arqueológica.

\section{Bibliografía}

ANDREAKI, V., BARCELÓ, J.A., BOGDANOVIC, I, GASSMAN, P., LÓPEZ BULTÓ, O., MORERA, N., PALOMO, A., PIQUÉ, R., REVELLES, J., TERRADAS, X., ANTOLÍN, F. 2020. Un modelo bayesiano para la cronología del yacimiento neolítico de La Draga (Banyoles, Girona). Un caso de estudio con ChronoModel 2.0. in J.A: BARCELÓ Y B. MORELL (eds., Métodos cronométricos en Arqueología, Historia y Paleontología, pp. 280-291. Madrid: Editorial Dextra.

ANTOLÍN, F., BUXÓ.R. 2011. L'explotació de les plantes al jaciment de la Draga: contribució a la històira de l'agricultura i de l'alimentació vegetal a Catalunya, in BOSCH, A., CHINCHILLA, J., TARRÚS, J (ed). El poblat lacustre neolític de la Draga: Excavacions de 2000-2005, monografies del CASC 9, 147-174.

BAENA, J., TERRADAS, X. 2005. ¿Por qué experimentar en Arqueología?”. Cursos sobre patrimonio Histórico Monografías de los Cursos de verano de Reinosa. Universidad de Cantabria, Reinosa, 141-160.

BUCH, M., COMELLAS, S., PALOMO, A. 2013 Arqueologia experimental: un recurs per l'aprenentatge significatiu i de descobriment in PALOMO, A; PIQUÉ, R; TERRADAS, X. (ed) Experimentación en arqueología. Estudio y difusión del pasado, Sèrie Monogràfica 25.1, 25.2., Museu d'Arquelogia de Catalunya, Generalitat de Catalunya, 35 - 42.

BOGDANOVIC, I; BOSCH; A; BUXÓ; R; CHINCHILLA. J; PALOMO, A; PIQUÉ, R; TERRADAS; X; TARRÚS, J. 2015. La Draga en el contexto de las evidencias de ocupación del lago de Banyoles in GONÇALVES, V.S.; DINIZ, M.; SOUSA, A. C., eds., V Congresso do Neolítico Peninsular. Actas. Lisboa: UNIARQ, 228-235.

BOSCH, A.; CHINCHILLA, J.; TARRÚS, J., 2006, Els objectes de fusta i fibres vegetals al poblat del neolític antic de la Draga (Banyoles, Pla de l'Estany), entre els anys 1995-2005, Monografies del CASC, núm.6. 
BOSCH, A.; PALOMO, A.; PIQUE, R.; TARRUS, J. 2006. Les fulles de pedra polida de les aixes in BOSCH, A.; CHINCHILLA, J.; TARRÚS, J. (ed), Els objectes de fusta del poblat neolític de la Draga, Excavacions 1995 - 2005, Monografies del CASC 9, 133-138.

BOSCH, A.; PALOMO, A., PIQUÉ, R., TARRÚS, J. 2008. Las láminas de piedra pulimentada y su relación con los mangos de azuela en el poblado de la Draga. A: HERNÁNDEZ M.S., SOLER, J.A., LÓPEZ J.A. (ed) IV Congreso del Neolítico Peninsular: 27-30 de noviembre de 2006., ed. Alacant. Museo Arqueológico de Alicante, 266 - 271.

BUCH, M, COMELLAS, S; PALOMO, A. 2011. El Parc Arqueològic del poblat neolític lacustre de la Draga. Una proposta de gestió patrimonial in $\mathrm{BOSCH}, \mathrm{A}$; CHINCHILLA, J; TARRÚS, J. El poblat lacustre del neolític antic de la Draga. Excavacions 1995-2005; Monografies del CASC 9, 229-236.

BUCH, M., COMELLAS, S., PALOMO, A. 2013 Arqueologia experimental: un recurs per l'aprenentatge significatiu i de descobriment PALOMO, A; PIQUÉ, R; TERRADAS, X. (ed) Experimentación en arqueología. Estudio y difusión del pasado, Sèrie Monogràfica 25.1, 25.2., Museu d'Arquelogia de Catalunya, Generalitat de Catalunya, 35 - 42.

CAMARÓS, E., SAÑA, M., BOSCH, A., PALOMO, A., TARRÚS, J. 2011. La arqueología experimental como instrumento para la interpretación de las herramientas en asta de la Draga (Banyoles, Catalunya) in MORGADO, A; BAENA, J; GARCÍA, D. (ed), La investigación experimental aplicada a la arqueología, Universidad Autónoma de Madrid, 239 - 244.

CLEMENTE, I., CUENCA, D. 2011. Instrumentos de trabajo de concha in BOSCH, A., CHINCHILLA, J., TARRÚS, J (ed), El poblat lacustre neolític de la Draga: Excavacions de 2000-2005, Monografies del CASC 9, 137-145.

Clemente, I., CUENCA, D., Oliva, M., ROSIllo, R., PAlOMO, A. 2014. Comparative analysis of Mytilus-shell implements from two Neolithic sites in NE Iberia: La Draga and Serra del Mas Bonet (Girona) In MARREIROS, L; BICHO, N; GIBAJA, J.F. (ed) International Conference on Use-Wear Analysis, 615 - 625. Algarve (Portugal).

DE DIEGO, M.; PIQUÉ, R.; PALOMO, A.; SAÑA,M.; TERRADAS, X.; CLEMENTE, I.; MOZOTA, M. 2018: Evidences of textile technology in the Ancient Neolithic site of la Draga. Some hypothesis, in SIENNICKA., RAHMSTORF, L., ULANOWSKA, A.(ed), First Textiles. The Beginnings of Textile Manufacture in Europe and the Mediterranean. Oxbow Books, Oxford, 69-80.

DE DIEGO, M., PAlOMO, A., PIQUÉ, R., ClEMENTE, I., TERRADAS, X. 2017.Traces of textile technology in the lacustrine early neolithic site of la Draga 
(Banyoles, Catalonia) from an experimental perspective in ALONSO, R., CANALES, D., BAENA, J. (ed), Playing with the time. Experimental archeology and the study of the past, 139-144, Servicio de Publicaciones de la Universidad Autónoma de Madrid

DE DIEGO, M.; PALOMO, A.; PIQUÉ, R.; SAÑA, M.; TERRADAS, X.; CLEMENTE, I.; MOZOTA, M. 2017: Fibre production and incipient textile technology in the Early Neolithic site of La Draga (Northeastern Spain, Banyoles-Catalonia; 53004900 BC) in BRAVERMANOVÁ, M., BŘEZINOVÁ, H., MALCOLM-DAVIES, J.(ed), Archaelological Textiles - Links between past and present. NESAT XIII, Liberec, pp. 293-302.

LÓPEZ-BULTÓ, O. 2015. Processos d'obtenció, transformació i ús de la fusta en l'assentament neolític antic de La Draga (5300-5000 cal ANE), PhD thesis, UAB

LÓPEZ-BUlTÓ, O; PIQUÉ; R., ANTOLÍN, F., BARCELÓ. J.A. PALOMO, A., CLEMENTE, I. 2020. Digging sticks and agriculture development at the ancient Neolithic site of la Draga (Banyoles, Spain). Journal of Archaeological Science: Reports. $\quad 30 \quad-\quad$ abril 2020, Elsevier, 01/04/2020. https://doi.org/10.1016/j.jasrep.2020.102193

LÓPEZ-BUlTÓ, O., PALOMO, A., CLEMENTE, I. On press. Tool mark analysis of neolithic wooden pointed sticks from la Draga (Banyoles, Spain), Quaternary International.

GIBAJA, J.F. 2000. La función del instrumental lítico tallado de la Draga (Banyoles, Pla de 1'Estany). In BOSCH, A., CHINCHILlA, J., TARRUS, J. (ed), El poblat lacustre neolític de la Draga. Excavacions de 1990-1998, Monografies del Casc 2, 206-213, Girona

GIBAJA, J.F., PALOMO, A. 2006, Les puntes de sageta y làmines de falç de sílex in BOSCH, A.; CHINCHILlA, J.; TARRÚS, J. (ed), Els objectes de fusta i fibres vegetals al poblat del neolitic antic de la Draga (Banyoles, Pla de l'Estany), entre els anys 1995-2005, Monografies del CASC, núm.6, Girona, 139-143.

GIBAJA, J.F., PALOMO, A., BICHO, N., TERRADAS, X. 2007. Tecnología y función de los "útiles astillados" in RAMOS, M.L., GONZÁLEZ, J.E., BAENA, J. (ed), Arqueología experimental en la Península Ibérica: investigación, didáctica y patrimonio, 157 - 164, Universidad Autónoma de Madrid.

GIBAJA, J.F., PALOMO, A., 2004. Geométricos usados como proyectiles. Implicaciones económicas, sociales e ideológicas en sociedades neolíticas del VI-IV milenio cal BC en el noreste de la Península Ibérica. Trabajos de Prehistoria, 61 - 1, pp. 81 97. Madrid, Comunidad de Madrid (España): CSIC- Instituto de Historia

GIBAJA, J.F., PALOMO, A., ARMENGOL, J. 2011. Aproximación experimental a la función de los proyectiles geométricos in MORGADO, A; BAENA, J; GARCÍA, D 
(ed), La investigación experimental aplicada a la arqueología, Universidad Autónoma de Madrid, 141 - 148, 2011

MOZOTA, M., PALOMO, A., GIBAJA, J.F., CLEMENTE, I. 2017. Experimental program: Neolithics awls and spatulas in ALONSO, R., CANALES, D., BAENA, J. (ed) Playing with the time. Experimental archeology and the study of the past, 61-66, Servicio de Publicaciones de la Universidad Autónoma de Madrid.

MOZOTA, M.; BORRELL, F.; CLEMENTE, I.; GIBAJA, J.F.; IBÁÑEZ, J.J.; NIETO, A.; ORTEGA, D.; TERRADAS, X.; VALENZUELA, S. 2018. Reference collections of the CSIC - Institute Milà i Fontanals for the study of prehistoric societies: Archaeology from an experimental and technological perspective in Experimental archaeology: from research to society -Proceedings of the Vth International Congress of Experimental Archaeology-, pp. 355-359. Tarragona: Reial Societat Arqueològica Tarraconense (Butlletí Arqueològic 40).

PALOMO, A. GIBAJA, J.F.; PIQUÉ, R., BOSCH, A.; CHINCHILLA, J.; TARRÚS, J. 2011a, Harvesting cereals and other plants in Neolithic Iberia: the assemblage from the lake settlement at La Draga, Antiquity. 85. p. 759 - 771

PALOMO, A.; PIQUÉ, R.; LÓPEZ-BULTÓ, O.; BOSCH, A.; CHINCHILLA, J.; TARRUS, J. 2011b. Análisis de los artefactos de madera del yacimiento neolítico lacustre de la draga. Aproximación experimental in MORGADO, A; BAENA, J; GARCÍA, D. (ed), La investigación experimental aplicada a la arqueología, Universidad Autónoma de Madrid, 245 - 253.

PALOMO, A. PIQUÉ, R., GIBAJA, J.F., LÓPEZ-BUlTÓ, O. BOSCH, A., CHINCHILLA, J. TARRÚS, J. 2011c. Tecnologia i funcionalitat dels objectes de fusta neolítics de La Draga una aproximació experimental in BOSCH, A., CHINCHILlA, J., TARRÚS, J (ed). El poblat lacustre neolític de la Draga: Excavacions de 2000-2005, monografies del CASC 9, p. 101 - 110.

PALOMO, A., CAMARÓS, E., GIBAJA, J.F. 2011d. La industria lítica i tallada. Una visió tècnica i experimental in BOSCH, A., CHINCHILLA, J., TARRÚS, J (ed). El poblat lacustre neolític de la Draga: Excavacions de 2000-2005, monografies del CASC 9, p. 79-89.

PALOMO, A. 2012. Tecnologia lítica $i$ de la fusta de la prehistòria recent al nord-est peninsular. Anàlisi tecnomorfològica $i$ experimental, $\mathrm{PhD}$ thesis, UAB.

PALOMO, A.; PIQUÉ, R.; TERRADAS, X.; LOPEZ, O.; CLEMENTE, I.; GIBAJA, J.F. 2013. Woodworking technology in the Early Neolithic site of La Draga (Banyoles, Spain) in ANDERSON, P., CHEVAL, C., DURAND, A (ed), Regards croisés sur les outils liés au travail des végétaux / An interdisciplinary focuson plant-working tools. 1 ed. Antibes. APDCAA, CPAM-CNRS; p. 383 - 396. 
PALOMO, A., PIQUÉ, R., TERRADAS, X. (ed). 2013, Experimentación en arqueología. Estudio y difusión del pasado, Sèrie Monogràfica 25.1, 25.2., Museu d'Arquelogia de Catalunya, Generalitat de Catalunya, 524p.

PALOMO, A.; PIQUÉ, R.; TERRADAS, X.; BOSCH, A.; BUXÓ, R.; CHINCHILLA, J.; SAÑA M.; TARRÚS, J. 2014. Prehistoric occupation in Banyoles lakeshore: results of recent excavations in La Draga site (Girona, Spain), Journal of Wetland Archaeology. 14.p. $58-73$

PALOMO, A., PIQUÉ, R., TERRADAS, X., BARCELÓ, J.A., RODRÍGUEZ, J.A., BUCH, M., JUNKMANNS, J., DE DIEGO, M., LÓPEZ-BULTÓ, O. 2017. Research, experimentation and outreach in the early Neolithic site of la Draga (Banyoles-Spain), EXARC JOURNAL Digest, 2017-2, 20-23

PIQUÉ, R; PALOMO, A; TERRADAS, X; TARRÚS, J; BUXÓ, R; BOSCH, A; CHINCHILLA, J; BOGDANOVIC, I; LÓPEZ-BULTÓ, O; SAÑA, M. 2015. Characterizing prehistoric archery: technical and functional analyses of the Neolithic bows from La Draga (NE Iberian Peninsula), Journal of Archaeological Science, Volume 55, March 2015, 166-173.

PIQUÉ, R., ROMERO, S. PALOMO, A., TARRÚS, J., TERRADAS, X., BOGDANOVIC, I. 2018. The production and use of cordage at the early Neolithic site of La Draga (Banyoles, Spain), Quaternary International, Volume 468, Part B, 25 February 2018, 262-270, https://doi.org/10.1016/j.quaint.2016.05.024

ROMERO BRUGUÉS, S, HERRERO OTAL, M., PIQUÉ, R. (on press) The basketry at the Early Neolithic site of la Draga (Banyoles, Spain) Journal of Archaeological Science Reports.

SAÑA, M. 2011. La gestió dels recursos animals in BOSCH, A., CHINCHILLA, J., TARRÚS, J (ed), El poblat lacustre neolític de la Draga: Excavacions de 20002005, monografies del CASC 9, 177-212. 